\title{
Hematoma retroperitoneal espontáneo. Caso clínico
}

\author{
Carlos Álvarez $\mathbf{Z}^{1,3}$, César Cerda Ca, Andrés Jadue $\mathrm{T}^{\mathrm{b}}$, \\ Francisco Rojas $\mathbf{R}^{\mathbf{b}}$, Millarai Abelleira $\mathbf{P C}$, \\ Carlos Hermansen $\mathrm{T}^{2,3}$, Francisco Gatica $\mathrm{J}^{1,3}$. \\ Spontaneous retroperitoneal \\ hematoma. Report of one case
}

\begin{abstract}
Spontaneous retroperitoneal hematoma is a rare condition. We report a 78 year-old man with progressive pain in his right thigh and hip lasting one week. The pain subsequently was associated with abdominal pain in the right lower quadrant. Physical examination revealed pain to deep palpation of the area, associated with a diffuse positive rebound pain. An abdominal and pelvis CT scan showed an extensive mesenteric hematoma. During surgery, a large retroperitoneal encapsulated hematoma, without evidence of active bleeding, was found and drained. Pathology confirmed the diagnosis and was negative for cancer (Rev Méd Chile 2007; 135: 1044-7).

(Key words: Hematoma; Mesenteric arteries; Radiography; Dual-energy Scanned projection)

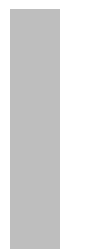

Recibido el 12 de julio, 2006. Aceptado el 30 de octubre, 2006.

${ }^{1}$ Departamento de Cirugía, Facultad de Ciencias Médicas, Universidad de Santiago de Chile. ${ }^{2}$ Departamento de Cirugía, Facultad de Medicina, Universidad de Chile. ${ }^{3}$ Unidad de Emergencia, Complejo Asistencial Barros Luco (CABL); Servicio de Cirugía CABL.

aAlumno de Medicina, Universidad de Santiago de Chile.

bInterno de Medicina, Universidad de Santiago de Chile.

'Médico Becario de Cirugía, Universidad de Chile. Santiago de Chile.
\end{abstract}

$\mathrm{E}^{1}$ hematoma retroperitoneal espontáneo (HRE) es una enfermedad rara, que se define como el hematoma del retroperitoneo o del mesenterio que ocurre sin historia reciente de trauma, tratamiento anticoagulante o enfermedad vascular ${ }^{1-3}$. Existen otras definiciones de HRE en la literatura, que hacen referencia a un hematoma que se produce por múltiples causas cuyo sangrado no está asociado al trauma ${ }^{4}$. Nos parece que esta definición no es la apropiada, ya que el término

Correspondencia a: Dr. Carlos Álvarez Zepeda. Gran Avenida 3204. San Miguel, Santiago, Chile. Fonos: 562-3948840. Fax: 562-5514320. E mail: doctormaz@gmail.com espontáneo se refiere a un sangrado producido sin causa determinada ni demostrable, tal como lo precisaron los autores japoneses ${ }^{1}$.

Para la revisión bibliográfica se realizó una búsqueda a través del Catálogo Bello, LILACS, PubMed y SciELO sin restricción de tiempo o idioma. Existen escasas comunicaciones de esta enfermedad en la literatura médica internacional y no las encontramos en la nacional.

\section{CASO CĹNICO}

Paciente de sexo masculino de 78 años de edad, con antecedente de alcoholismo desde hacía 10 años. Ingresó a la Unidad de Emergencia del 
Complejo Asistencial Barros Luco (CABL) con historia de una semana de evolución, caracterizada por dolor en muslo y cadera derecha, progresivo en intensidad. El día de su ingreso al hospital se asoció a dolor abdominal en el cuadrante inferior ipsilateral y a un episodio de lipotimia. No presentaba antecedentes de trauma abdominal ni otras enfermedades asociadas. En el examen físico de ingreso destacaba una presión arterial de 80/54 mmHg, pulso 78 x', coloración lívida de la zona infraumbilical de la pared abdominal y de las extremidades inferiores. Se aportaron entonces 1.000 cc de solución cristaloide intravenosa que permitieron normalizar la presión arterial. El abdomen aparecía distendido, con dolor intenso a la palpación superficial y profunda en hipocondrio, flanco y fosa ilíaca derecha. Presentaba, además, dolor de rebote difuso, resistencia muscular difusa y puño percusión derecha positiva. En los exámenes de laboratorio de ingreso se obtuvo: glicemia de $250 \mathrm{mg} / \mathrm{dl}$, nitrógeno ureico de $17 \mathrm{mg} / \mathrm{dl}$, creatininemia de $1,17 \mathrm{mg} / \mathrm{dl}$, amilasa $86 \mathrm{U} / \mathrm{l}$, lipasa $40 \mathrm{U} / \mathrm{l}$, proteína $\mathrm{C}$ reactiva $2 \mathrm{mg} / \mathrm{l}$, protrombinemia 83,5\%; tiempo de tromboplastina parcial activado: $28,1 \mathrm{~s}$, recuento de leucocitos de 18.200/ul, hemoglobinemia de $12,7 \mathrm{mg} / \mathrm{dl}$ y un hematocrito de 39\%. Se realizó una tomografía computada (TC) de abdomen y pelvis, con contraste, que mostró una masa espontáneamente hiperdensa a nivel del hipocondrio y flanco derecho de 16 por 12 por $9 \mathrm{~cm}$ y que desplazaba el colon derecho hacia la periferia, manteniendo un plano de clivaje con él. Se sugería la presencia de un extenso hematoma mesentérico, sin poder descartar también neoplasia subyacente (Figuras 1 y 2). Al segundo día de hospitalización el paciente estaba hemodinámicamente estable, afebril y con menos dolor abdominal espontáneo, sin embargo, el hematocrito de control descendió a $21 \%$, decidiéndose entonces transfundir $2 \mathrm{U}$ de glóbulos rojos e intervenir quirúrgicamente al paciente.

Se efectuó laparotomía exploradora, encontrándose un gran hematoma encapsulado retroperitoneal derecho, que se extendía desde el mesocolon transverso hasta la pelvis, sin observar lesión con sangrado activo. Se realizó vaciamiento, aseo, instalación de drenajes en el retroperitoneo y toma de muestras para biopsia, la que, posteriormente, se informó como hematoma de mesenterio sin evidencias de neoplasia.
El paciente evolucionó satisfactoriamente hasta el sexto día del posoperatorio, cuando se observó salida de líquido fecal por los drenajes, realizándose el diagnóstico de fístula estercorácea. Se decidió, entonces, conectar los tubulares a aspiración e iniciar tratamiento antibiótico con metronidazol más ciprofloxacina, con lo que se consiguió buen control de la fístula, la cual evolucionó al cierre definitivo al vigésimo día desde la opera-

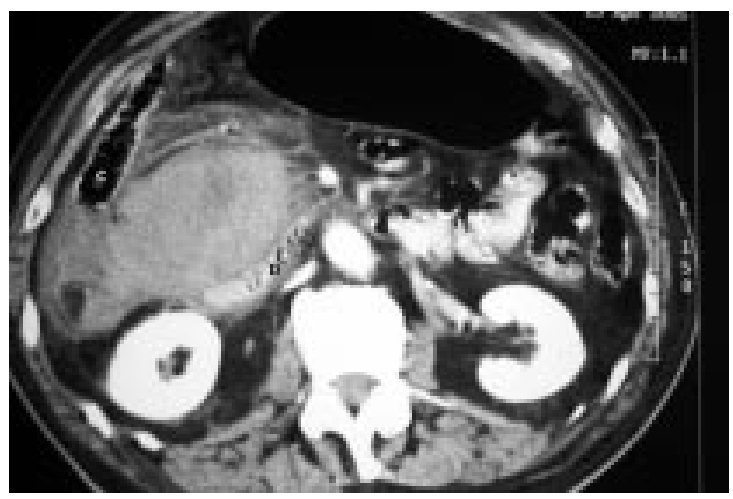

Figura 1. TC de abdomen con contraste endovenoso a nivel del tercio medio del ninón derecho. Se observa una masa retroperitoneal hiperdensa, alojada en la raíz del mesenterio con extensión al espacio pararrenal derecho, con contraste hidrosoluble en su interior disperso de manera amorfa. La masa desplaza el colon derecho (C) y la segunda y tercera porción del duodeno (D).

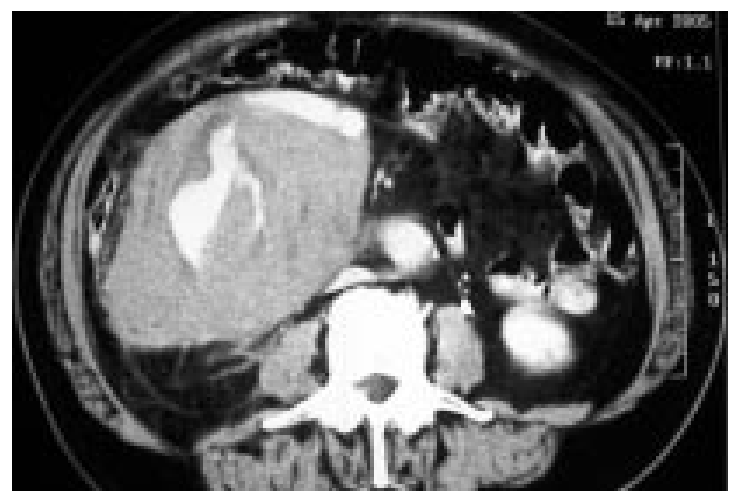

Figura 2. TC de abdomen con contraste endovenoso a distal al polo inferior del riñón derecho. Se observa una gran masa retroperitoneal hiperdensa heterogénea con áreas líquidas y otras zonas con presencia de contraste hidrosoluble disperso de manera amorfa en su interior. 
ción, dándose de alta en óptimas condiciones generales y de la herida. El paciente se ha mantenido en control ambulatorio en policlínico de cirugía, permaneciendo asintomático a 6 meses de su egreso.

\section{DISCUSIÓN}

Los casos de HRE son escasos en la literatura médica ${ }^{1-3,5-7}$. En Chile no encontramos casos publicados. El paciente que presentamos cumple con los requisitos enunciados de ausencia de trauma abdominal reciente, tratamiento anticoagulante o enfermedad vascular $\mathrm{y}$, al igual que la mayoría de los casos, presentaba importante dolor abdominal acompañado de anemia ${ }^{1}$. Sin embargo, destaca en este paciente, que a pesar de ingresar hipotenso, se logró posteriormente su estabilidad hemodinámica, lo que lo diferencia de la mayoría de las otras comunicaciones donde es más frecuente la persistencia de hipotensión y choque ${ }^{2}$.

En cuanto a la causa de esta enfermedad, ella permanece aún incierta ${ }^{2,3}$. No obstante, se ha encontrado que existen varios factores asociados, como la hipertensión arterial, la arteriosclerosis, las malformaciones arteriales y las diátesis hemorágicas ${ }^{1,3,8}$. En nuestro paciente no encontramos evidencia cierta de ninguno de ellos. Su carácter espontáneo queda confirmado al reunir los hallazgos clínicos preoperatorios, imagenológicos, intraoperatorios, histológicos y el curso benigno de su posoperatorio y controles posteriores.

El dolor de muslo y cadera derecho que presentaba este caso, es homologable a la sintomatología de paresia y dolor de neuropatía femorocrural que en la literatura aparece hasta en $60 \%$ de los hematomas retroperitoneales ${ }^{9,10}$.

La situación clínica esperable frente a la acumulación de sangre en el retroperitoneo es la presencia de masa palpable, dolor, hipotensión y una caída en el hematocrito, estos tres últimos están presentes en el caso que se comunica.

El diagnóstico de esta patología depende principalmente de las imágenes y los hallazgos intraoperatorios. La TC y la ecografía son las herramientas más utilizadas $2,8,11$. Dentro de las primeras dos semanas, la mayoría de los hematomas son hiperdensos cuando se los compara con el tejido circundante, que fue lo que se pudo observar en nuestro caso. En la medida que el hematoma va madurando, su densidad va disminuyendo progresivamente para llegar a ser una masa de tejido blando. Varias semanas después, la atenuación radiológica puede llegar a ser igual al nivel del suero, simulando una lesión quística ${ }^{1}$. Con el tiempo va creciendo una membrana fibrovascular alrededor del hematoma, produciendo la imagen de un denso anillo en la $\mathrm{TC}^{1,2,10,13}$. La resonancia magnética (RM) tiene poco valor para el diagnóstico de coágulos frescos, pero puede jugar un rol importante en la determinación de los hematomas antiguos ${ }^{2}$. En nuestro caso, al parecer, la RM no hubiese tenido mayor utilidad.

Dentro de los diagnósticos diferenciales del HRE están los tumores y las masas quísticas del retroperitoneo. Los tumores retroperitoneales son poco frecuentes, representan entre $0,3 \%$ y $0,8 \%$ de todas las neoplasias ${ }^{14}$. Entre los tumores, el liposarcoma es el tumor maligno retroperitoneal más frecuente. Otros tumores a considerar son el fibrohistiocitoma, los schwannomas y los paragangliomas ${ }^{15-17}$. Las lesiones quísticas del retroperitoneo pueden ser neoplásicas o no neoplásicas. Las neoplásicas incluyen una variedad amplia de lesiones donde están el linfangioma quístico, el cistoadenoma mucinoso y el teratoma quístico entre otras. Las no neoplásicas incluyen al pseudoquiste de páncreas, el linfocele y el urinoma. Aunque los hallazgos clínicos e imagenológicos pueden ser a veces similares entre estas lesiones, algunas características de la TC, como la localización, el tamaño, la forma, las características de la pared, la presencia de tabiques o calcificaciones y el compromiso de las estructuras adyacentes pueden sugerir en algunas ocasiones el diagnóstico específico ${ }^{18}$.

El manejo de los HRE depende de la presentación clínica. Se indica la cirugía de urgencia si el paciente muestra signos de una importante pérdida de sangre o una complicación secundaria, tal como una obstrucción o una isquemia intestinal. $\mathrm{Si}$ los hematomas retroperitoneales son pequeños, estables y oligosintomáticos, podrían no requerir cirugía y evolucionar espontáneamente ${ }^{1}$. Cuando se realiza cirugía, el procedimiento incluye la disección y evacuación del hematoma y la búsqueda de un punto sangrante. Cuando resulta posible la ubicación de una lesión responsable de 
la hemorragia, el caso ya no es por definición un HRE y, por ejemplo, en la eventualidad de sangrado renal habitualmente conlleva a una nefrectomía ${ }^{2,4}$. En nuestro caso hubo un importante descenso del hematocrito y, además, no fue posible una certeza diagnóstica preoperatoria que descartase una neoplasia o una enfermedad vascular. La intervención y la biopsia aclararon el diagnóstico definitivo de $\mathrm{HRE} \mathrm{y}$, si bien, se

\section{REFERENCIAS}

1. Aoki T, Nishizono M, Nina $H$, Inatsu $H$, Komidori H, Itano T ET aL. A case of spontaneous mesenterio hematoma and a review of 17 cases in Japan. Gastroenterol Jpn 1990; 25: 768-73.

2. Ming Ch, Hongwei X, Guangzhao Y, Bin Y. Spontaneous hematoma in the root of the small bowel mesentery: imaging findings. Chin Medical J 2003; 116: 954-6.

3. Zhu DY, Cheng YQ, Xue RC. Abdominal apoplexy: report of 3 cases. Chin J Dig 1982; 2: 173-4.

4. Pérez MA, Caeiro S, Cachaldora Ja, Cal L, Vidal J, Segura R. Hematoma retroperitoneal espontáneo como causa de dolor abdominal y shock hemorágico. Angiología 1998; 50: 95-8.

5. OKaZaKi M, KовaYashi K, YамамоTO S. A rare case of intraabdominal bleeding due to ruptured mesenteric hematoma. Jpn J Soc Clin Surg 1978; 39: 242.

6. Sugano S, Fukukawa K, Miyairi $Y$, Aikawa $K$, Abei $T$. A case of spontaneous mesenteric hematoma. Jpn J Clin Radiol 1986; 31: 331-4.

7. Singla SK, Champakam NS, Bishnoi PK, Bajaj P, CHOpRA S. Spontaneous mesenteric hematoma. Indian J gastroenterol 1989; 8: 120-1.

8. Raghavendra BN, Grieco AJ, Balthazar EJ, Megibow AJ, SubRamanyam BR. Diagnostic utility of sonography and computed tomography in spontaneous mesenteric hematoma. Am J Gastroenterol 1982; 77: 570-3.

9. Codina Cazador A, Ruiz Feluu B, Roig García J, tuca Rodríguez F, Farres Coll R, Masvidal Calpe R. Hematoma retroperitoneal secundario a tratamiento anticoagulante. Rev Esp Enf Digest 1993; 83: 133-5.

10. Gómez de la Torre R, Mila Crespo A, Cadenas F, Fernández Bustamante $\mathrm{J}$, Vázquez Castañón $\mathrm{M}$. presentó una fístula estercorácea posoperatoria, probablemente consecuencia de la disección del colon derecho al liberar el hematoma, ésta fue manejada en forma conservadora, con éxito.

En conclusión, el HRE debe considerarse en el diagnóstico diferencial de las masas del retroperitoneo. La familiaridad con los hallazgos clínicos e imagenológicos de las masas retroperitoneales facilita el acertado diagnóstico y tratamiento.

Hematoma retroperitoneal espontáneo inducido por enoxiparina a dosis terapéuticas. An Med Interna 2003; 20: 386-7.

11. Fon GT, Hunter TB, Haber K. Utility of ultrasound for diagnosis of mesenteric hematoma. AJR 1980; 134: 381-4.

12. New PFJ, Davis KR, Ballantine HT. Attenuation measurements of whole blod and blood fractios in computed tomography. Radiology 1976; 121: 625-40.

13. Josepth KTL, Stuart SS, Robert JS. Computed body tomography with MRI correlation. $2^{\text {nd }}$ Ed. Raven Press, New York. 1989: 661-4.

14. Álvarez T, Gómez E, Guevara B, Soriano J, Carrera E, DuRán M. Tumores retroperitoneales. Revisión de cinco años en material de autopsia. Rev Med Hosp Gen Mex 2004; 67: 78-82.

15. Hasegawa T, Seiki K, Hasegawa F, Matsuno $Y$, Shimodo T, Hirose T et al. Dedifferentiated liposarcoma of retroperitoneum and mesentery: varied growth patterns and histological grades: a clinicopathologic study of 32 cases. Hum Pathol 2000; 31: 717-27.

16. Ghal VS, Gold JE, Vincent RA, Cosgrove JM. Malignant peripheral nerve sheath tumor arising spontaneously from retroperitoneal ganglioneuroma: a case report, review of the literature, and immunohistochemical study. Hum Pathol 1992; 23: 72-5.

17. Lack EE, Cubila AL, Woodruff JM, Lieberman PH. Extraadrenal paragangliomas of the retroperitoneum: a clinicopathologic study of 12 tumors. Am J Surg Pathol 1980 Apr; 4: 109-20.

18. Yang DM, Fung DH, Kim H, Kang FH, Kim SH, Kim FH ET AL. Retroperitoneal Cystic Masses: CT, Clinical, and Pathologic Findings and Literature Review. RadioGraphics 2004; 24: 1353-65. 